Moroccan J. of Pure and Appl. Anal. (MJPAA)

Volume 8(1), 2022, Pages 78-91

ISSN: Online 2351-8227 - Print 2605-6364

DOI: $10.2478 / \mathrm{mjpaa}-2022-0007$

\title{
Pricing American Put Option using RBF-NN: New Simulation of Black-Scholes
}

\author{
El Kharrazi Zaineb ${ }^{1}$, SAOUd SAHAR ${ }^{2}$, Mahani Zouhir ${ }^{3}$
}

\begin{abstract}
Aвstract. The present work proposes an Artificial Neural Network framework for calculating the price and delta hedging of American put option. We consider a sequence of Radial Basis function Neural Network, where each network learns the difference of the price function according to the Gaussian basis function. Based on Black Scholes partial differential equation, we improve the superiority of Artificial Neural Network by comparing the performance and the results achieved used in classic Monte Carlo Least Square simulation with those obtained by Neural networks in one dimension. Thus, numerical result shows that the Artificial Neural Network solver can reduce the computing time significantly as well as the error training.

Mathematics Subject Classification (2020). 91G20, 92B20, 65C05, 91G80

Key words and phrases.American option, Artificial neural network, Black-Scholes equation, Monte Carlo simulation, Option pricing, Radial basis function
\end{abstract}

\section{Introduction}

Derivatives have become more and more popular and essential in the finance world [17, 41]. Options, for example, are one of the most basic and important derivatives that actively traded on many exchanges. For that, the option valuation receives high attention. The holder of the option can sell or buy the underlying asset, for example, a quantity of a company's stock, at

Received December 21, 2020 - Accepted: June 17, 2021.

(C) The Author(s) 2021. This article is published with open access by Sidi Mohamed Ben Abdallah University.

${ }^{1}$ Engineering Sciences and Energy Management Laboratory, ENSA, Ibn Zohr University

zaineb.elkharrazi@edu.uiz.ac.ma (Corresponding Author)

${ }^{2}$ Technical Research Laboratory, Faculty of Applied Sciences, Ibn Zohr University

sahar.saoud@gmail.com

${ }^{3}$ Engineering Sciences and Energy Management Laboratory, ENSA, Ibn Zohr University

zouhir.mahani@gmail.com. 
predetermined expiration date and a given price fixed before in the contract [27, 31, 35]. A put (call), option gives the owner the right to sell (buy) the underlying asset, and the price option differs according to the types of options: European options, American options, Asian options, Barrier options, Bermudan options... etc. Unlike the European option, which can be exercised just at maturity, American options can be exercised at any time until maturity, hence the valuation of this type of option does not have a close form solution.

Therefore, for the valuation of financial derivatives and in modern risk management, numerical methods are used. Generally speaking, in financial mathematics, there are two main approaches to calculating price option: either we use the risk-neutral probabilities to compute the expected asset values, or we obtain the price of the option using the Black-Scholes equation $[6,14]$. However, these approaches are connected by the classical Feynman Kac theorem [33], which provides that the classical solution of a linear parabolic partial differential equation has a stochastic representation in terms of an expected value [14, 16].

Moreover, Black Scholes model was developed by Fisher Black, Robert Merton and Myron Scholes in 1973 [6], proposed the famous Black Scholes formula for pricing options, which is considered as one of the best ways of determining accurate prices of options, and still widely used in financial markets today. As a result, 1973 is considered as the beginning of a period of intensive research into the valuation of derivative products. During the $80 \mathrm{~s}$ and $90 \mathrm{~s}$, the hedging of these products made market actors aware of the risk [36, 43].

To improve adequacy and performance, three major families of computational approaches based on the Black Scholes model have been studied: Binomial Trees, Finite Difference methods, and Monte Carlo methods [21, 22, 45]. Moreover, artificial neural networks have recently gained much attention due to their success in a number of different fields and applications, including finance and especially pricing options [24, 28]. Artificial neural networks are information treatment models that have developed as generalizations of mathematical models of human knowledge, inspired by the working principles of the human brain [1,12, 23]. The basic component of an artificial neural network is neuron (ex: like biological neuron). A biological neuron may be modeled artificially to perform computation, and then the model is characterized as an artificial neuron. A neuron is the basic processing element in a neural network [47]. Each neuron receives one or more inputs to produce output.

In this article, we adapt the RBF-NN into the pricing American option problem in one dimension, and then we compare this approach with the Least Square Monte Carlo method. The main objective of this work is to show that the use of neural network, leads to refinement and a sophisticated model which will be a useful tool to study the problem of pricing contingent claims in finance. So, in section 2, we described the Black Scholes model involving Monte Carlo approaches and the finite difference method. In section 3, we described our approaches to price the put option using RBF-NN combined with the finite difference method. Finally, section 4 is dedicated to numerical results and where we show the performance and sensitivity, that we calculated using delta hedging, of the proposed method. 


\section{Option Pricing using Black-Scholes model}

The Black-Scholes equation and the associated Black-Scholes partial differential equation have been discussed in the work of Black and Scholes (1973), is one of the most well-known results in quantitative finance $[2,35]$. It is used to calculate the value of various financial derivatives, such as American options.In this section, two approaches are briefly presented to evaluate the American put option: Monte Carlo simulation and finite difference method.

2.1. Black-Scholes Model. According to the Black-Scholes model, the dynamics of a risky asset follow a stochastic differential equation (SDE). We consider an American put option with maturity $T>0$, and strike $K>0$ written on an underlying asset $S$.

Let $W=\left(W_{t}\right)_{t \geqslant 0}$ be the standard Brownian motion started at time $t_{0}=0$. We consider $S=\left(S_{t}\right)_{t \geqslant 0}$ to be the geometric Brownian motion that governs the price of the asset in the market. In addition, $\sigma>0$ and $r>0$ is the volatility coefficient and interest rate respectively. Then the stock price process satisfies:

$$
d S_{t}=r S_{t} d t+\sigma S_{t} d W_{t}, \quad S_{0}=s>0
$$

Using the Itô's formula [33], the solution of SDE (1) is given by:

$$
S_{t}=S_{0} \exp \left[\left(r-\frac{\sigma^{2}}{2}\right) t+\sigma W_{t}\right]
$$

To describe the American option pricing problem, let $\mathcal{T}[t ; T]$ be the set of admissible stopping times in $[t ; T]$ and $\tau \in \mathcal{T}[t ; T]$ is a stooping time, and let $\mathbb{Q}$ be the risk-neutral measure ${ }^{1}$. Then, the American put option price is given by:

$$
V(t, s)=\sup _{\tau \in \mathcal{T}[t ; T]} \mathbb{E}^{Q}\left[e^{-r(\tau-t)} G\left(S_{\tau}\right) \mid S_{t}=s\right]
$$

With $G$ is the payoff of the put option:

$$
G\left(S_{\tau}\right)=\left(K-S_{\tau}\right)^{+}
$$

(2.3) is a dynamic programming equation close to the equations which allows to calculate $\mathbb{E}\left(G\left(S_{T}\right)\right)$, and $u(t, s)=\sup _{t \leqslant \tau \leqslant T} \mathbb{E}\left(G\left(\tau, S_{\tau}\right) \mid S_{t}=s\right)$

Otherwise, in $t$, if $S_{t}=s$, either we exercise in $t$ and then we win $G(t, s)$, or we wait until $t+1$ where we can win $u\left(\left(t+1, S_{t+1}\right)\right.$.

Using dynamic programming arguments, the optimal stopping problems are expressed by a dynamic programming equation [34], where its solution is equivalent to the American option price. Under some regularity assumptions, $V$ is the unique solution of the following partial differential equation:

$$
\begin{aligned}
\partial_{t} V+r S \partial_{s} V+\frac{1}{2} \sigma^{2} S^{2} \partial_{s S} V-r V & =0 & & \{(t, s), V(t, s)>G(s)\} \\
V(t, s) & \geqslant G(s) & & (t, s) \in[0, T] \times \mathbb{R}
\end{aligned}
$$

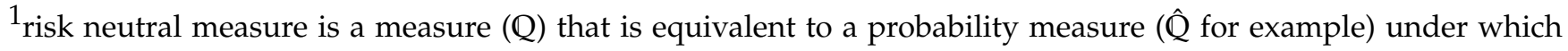
the discounted stock price process is a martingale 


$$
V(T, s)=G(s) \quad s \in \mathbb{R}
$$

Next, we choose to introduce two famous methods: Least Square Monte Carlo and Finite Difference methods, which aim to solve Black Scholes partial differential equation (2.5).

2.2. Least Square Monte Carlo Valuation. Generally speaking; the Monte Carlo simulation techniques use the strong law of large numbers to estimate the value sought [37]. If the curves of $S^{i}=\left\{S_{i}^{t}, 0 \leqslant t \leqslant T\right\}, 1 \leqslant i \leqslant N$, represented $N$ independent distributed trajectories according to the same law, then:

$$
G=\frac{1}{N} \sum_{i=1}^{N} G\left(S^{i}\right) \rightarrow g^{\prime} \text { p.s. }
$$

The basic idea of the Monte Carlo methods is to generate $N$ trajectories $S^{i}=\left\{S_{i}^{t}, 0 \leqslant t \leqslant T\right\}$, and to consider $G$ as a price stock estimator, which converges to $G^{\prime}$, then the central limit theorem allows having an idea about the achieved approximation. In other words, the Least Square Monte Carlo method utilizes the fact that we know the simulated continuation value at time $t$ for $i^{\text {th }}$ path but can't use it directly.

Besides, the American put option price depends on the optimal stopping time $\tau$ given by equation (2.3), where the expectation is taken under the risk-neutral measure $\mathbb{Q}$. Longstaff Schwartz's algorithm [27] consists of an approximation of the optimal stopping time $\tau$ on $N$ trajectories, by the random variables $\tau^{i}$, which depends on the $i^{\text {th }}$ trajectory. Using the Markov property of $S_{t}$, to calculate the option value at time $t$ as $V_{t}$, the continuation value at time $t$ is then approximated using a Monte Carlo method [25, 45].

Algorithm 1: Least square Monte Carlo method

- Divide the time interval into a subdivision interval $t_{0}=0, t_{1}, \ldots, t_{N}=T$,

- Simulate $M$ random trajectories for $S$ in a risk-neutral world,

- Calculate derivative payoff obtained from the Black Scholes formula:

$S_{t}=S_{0} \exp \left[\left(r-\frac{\sigma^{2}}{2}\right) t+\sigma \sqrt{T} Z\right]$, with $Z$ a random variable,

- Compute the payoff at maturity for each trajectory $\left(k-S_{N}^{i}\right)^{+}$,

- Going backward from maturity and repeating the previous step in order

to collect many sample values of the payoff,

- Regress the discounted continuous value according to $S$ at current time using the Monte Carlo estimator:

$$
\text { Put }_{0} \approx \exp ^{-r t} \frac{1}{M} \sum_{M} \text { payoff }
$$

2.3. Black Scholes Equation and Finite Difference. Finite Difference is one of several studies or methods that appear to solve accurately the famous Black Scholes partial differential equation $[8,19]$. In 1911, Richardson proposed the Finite Difference approximations for numerically solving partial differential equations; then the Finite Difference method was applied to option 
pricing for the first time by Eduardo Schwartz in 1977[38].

To establish the link between the Black-Scholes and the heat equation, we consider the classic heat equation in one dimension

The terms $-r V$ and $r S$ in Black Scholes equation (2.5), significantly modify the change of variables which transform this equation in the heat equation. We pose:

So

$$
S=\operatorname{Kexp}(x), \quad t=T-\frac{2 d}{\sigma^{2}}, \quad q=\frac{2 r}{\sigma^{2}}
$$

$$
V(S, t)=V\left(\operatorname{Kexp}(x), T-\frac{2 d}{\sigma^{2}}\right)=v(x, d)
$$

Then

$$
v(x, d)=\operatorname{Kexp}\left(-\frac{1}{2}(q-1) x-\frac{1}{4}(q+1)^{2} d\right) u(x, d)
$$

with $u$ is the solution of the heat equation

$$
\left\{\begin{array}{l}
\frac{\partial u}{\partial d}-\frac{\partial^{2} u}{\partial x^{2}}=0 \\
u(d=0, x)=u_{0}(x)
\end{array}\right.
$$

Applying the finite difference approximations on the derivatives in (2.7), gives the following approximations of the derivatives:

$$
\begin{gathered}
\frac{\partial u}{\partial d}=\frac{u_{i+1, j}-u_{i-1, j}}{2 \delta d} \\
\frac{\partial^{2} u}{\partial x^{2}}=\frac{u_{i, j+1}-2 u_{i, j}+u_{i, j-1}}{(\delta x)^{2}}
\end{gathered}
$$

We divide the time interval into $i$ steps, and $\delta d$ is the distance between each step. In the same way, we divide the interval of $x$ into $j$ steps, then $\delta d$ represent the distance between each step.

The main idea behind the finite difference is to approximate the differential equation with different operators in order to estimate and discretize the unknown function in the differential equation $[2,8,19]$.

\section{Pricing American option using Neural Network Radial Basis Function}

3.1. Machine Learning and Neural Network. Most introductory texts bring up brain analogies when describing neural networks. Thus, neural networks are one of several approaches used in machine learning that has received huge attention in the last few years due to their success in many real-life problems such as image recognition, medical diagnostic, finance, etc. Neural Networks are essentially a part of Machine Learning, which refers to computer algorithms that learn from data or automate data based on model building and programming through the pre-information and the associated performance measure in the available data.[35, 29, 47], 


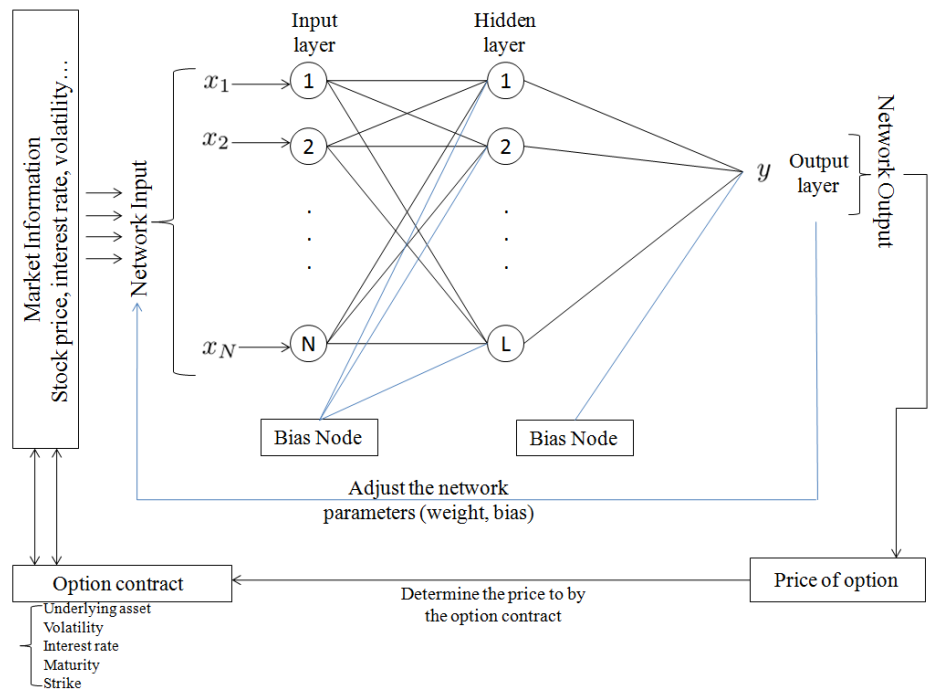

FIGURE 1. Financial Market and Artificial neural network architecture

In this paper, we try to use neural network to accelerate or speed up the valuation of our option by learning the results of an option pricing method, and to minimize the training error in order to detect the exact price of our option; avoiding underpricing or overpricing the option. From a computational point, the artificial neural network does not have a big impact on the high dimensionality of partial differential equations, which consider as an important advantage of this method, yet in our work, we still work in one dimension until we verify at first the efficiency of neural network in one dimension.

Neural network relates a set of input variables $\left\{x_{i}\right\}, i=1, \ldots, N$, to a set of output variables (it could be one output or more), $\left\{y_{j}\right\}, j=1, \ldots, k$.

As shown in Figure (1), artificial neural network is composed of three steps: neurons, layers, and the architecture that combines different layers.

The artificial neuron is based on some principal steps and operations:

Step 1: Calculate the summation of weighted inputs

Each input $x_{i}, i=1, \ldots, N$, is weighted before achieving the neuron by weight factor $w_{i j}$ for $j=1, \ldots, L$, so we calculate this summation:

$$
\sum_{j=1}^{n} w_{i j} x_{j}
$$

Step 2: Add the bias to the sum

Each neuron has a bias term $w_{0}$, which has to be achieved or exceeded for the neuron to produce an output signal (price option in our case). 
Step 3: Compute the output using an activation function

To determine the output signal, each neuron applies a mathematic function called activation function to its net input.

Let's consider a function $f(s)$, this function acts on the produced weighted signal, Mathematically, the output of the $i^{\text {th }}$ neuron $N_{i}$ is:

$$
y_{i}=f\left[w_{o}+\sum_{j=1}^{n} w_{i j} x_{j}\right]
$$

Several approaches have been used to approximate the Black-Scholes model for evaluating the performance and the values obtained from the Black-Scholes equation.

The aim of our work is to use neural network, more precisely, we will use the neural network radial basic function to evaluate the price of American put option, and then we will compare the results with the famous Monte Carlo method in the next.

3.2. Neural Network-Radial Basis Function for pricing american option. The basic idea here is to relate the Black-Scholes model with machine learning from an optimization point of view. To estimate the model parameters, we have to find the value of neural networks hidden units, so that output matches the observed option prices.

Generally, the popular neural networks used in function approximation are Multilayer Layer Perceptron (MLP) and Radial Basis Function (RBF) networks, here we concentrate on RBF-NN.

RBF networks, in principle, could be employed in linear or non-linear models, and in any sort of network (single layer or multi-layer), even if the renowned utilization of the basis function is in a single layer. Aptly, since Broomhead and Lowe's 1988 seminal paper, RBF networks have been traditionally associated with radial functions in a single layer network such as shown in figure (1).

Our choice is based on the fact that in the RBF network it is essential to set correct initial states; while MLP networks use randomly generated parameters initially, and since our input are not random because we obtained them from finite difference result, it seems that RBF neural network is more appropriate in our case.

Otherwise, the inputs used in the RBF network are the output of the finite difference method, which means that we use the finite difference in order to calculate the price of American put option, then the results obtained from this method become the input of the RBF-NN so that the input vector used is not random.

3.2.1. Neural Networks Radial Basis Function. In neural RBF, the distance between the input vector and the prototype vector determines the activation unit. This class of neural network has its roots in techniques for performing an exact approximation of data points in a multidimensional space [46].

The input can be modeled as a vector of real numbers. Or, the radial basis function network approach introduces a set of $n$ basis function, one for each input or data point, and each node 
in the hidden layer represents a Gaussian ${ }^{2}$ basis function which take the form $\phi\left(\left\|x-x^{n}\right\|\right)$ where $\phi(x)$ is a non-linear function represented as follow:

$$
\phi(x)=\exp \left(-\frac{\left(x-c_{i}\right)^{2}}{2 \sigma^{2}}\right)
$$

we have $x$ is the input vector, $c_{i}$ the centre of the neuron, and $\sigma$ is the width Thus, the $\mathrm{n}^{\text {th }}$ function depends on the distance $\left\|x-x^{n}\right\|$, between $x$ and $x^{n}$. Then the output is given by a linear combination of the basis functions:

$$
f(x)=\sum_{i=1}^{n} w_{i} \phi_{i}\left(\left\|x-x^{i}\right\|\right)
$$

Where $w_{i}$ represents the weight of the layer, $\phi$ is the basis function and $n$ is the number of RBF (hidden layer).

Weight values connect between the hidden and output layers are found by solving the following system:

$$
w_{i}=\left(\phi^{t} \phi\right)^{-1} \phi^{t} t_{i}
$$

where $t_{i}$ is a column vector of target outputs, and where $\phi^{t}$ is the transpose of matrix $\phi$. The exact interpolation problem required every input vector to be mapped exactly into the corresponding target vector [18].

3.2.2. Finite Difference Radial Basis Function Networks. We choose in this paper to combine the Finite Difference method with neural network RBF, in order to find the price of our put option in the shortest time and with a small error. The key of these combined methods is that the output of finite difference becomes the input of RBF networks, to get the optimal weight and bias.

Furthermore, to clarify the mechanism of our method, we can distinguish between three steps since the radial basis function consists of three layers:

The first layer is the input layer, which is a vector of price values obtained from finite difference (in section 2) and which only transfers the data to the next layer.

The second layer is a hidden layer with a non-linear RBF (we use Gaussian function), where each input neuron is connected to all hidden layer neurons, and each neuron consists of a radial basis function centered on a point. the centres $c_{i}$, the widths $\sigma_{i}$ and the weights $w_{i}$ are simultaneously adjusted in order to minimize the least error square cost function and they are determined by the training process. Then, a hidden neuron computes the Euclidean distance of the test case from the neurons center point and then applies the RBF function to this distance. The resulting value is passed to the summation layer.

The third layer is a linear output layer. Its output is given by equation (3.1), as we can see the value coming out of a neuron in the hidden layer is multiplied by a weight associated with

${ }^{2}$ There are different types of basis function but in this work, we choose Gaussian basis function 
the neuron and passed to the summation which adds up the weighted values and presents this sum as the output of the network.

The architecture of our proposed method, RBF neural network, in the training and testing stage is given by the following algorithm:

Algorithm 2: Radial Basis Function Neural Network

- Initialize the parameters of Black Scholes equation $r, T, K, S_{0}, S_{\text {max }}$,

$S_{\text {min }}, \sigma$

- Calculate initial conditions $\log \left(\frac{S_{\min }}{K}\right), \log \left(\frac{S_{\max }}{K}\right)$ and semi

analytic solution, taking on consideration the boundary condition

$$
\left\{\begin{array}{l}
V(t, s) \geqslant G(s) \quad(t, s) \in[0, T] \times \mathbb{R} \\
V(T, s)=G(s) \quad s \in \mathbb{R}
\end{array}\right.
$$

- By Finite Difference method calculate the results $u$ of each level in the

Black Scholes equation

- Set $u$ as input and semi analytic solution as target vector of neural

network

- Initialize RBF-NN parameters (weight and bias) randomly

- Training input data by using RBF-NN to find the optimal

weight and bias

- Testing: Simulate the input $u$ data with target by using optimal weight and bias

After initializing the knowing parameters of Black Scholes model, we calculate the initial conditions $\log \left(\frac{S_{\max }}{K}\right)$ and $\log \left(\frac{S_{\min }}{K}\right)$, that we get from change of variable, and which we use to link the Black Scholes equation with the heat equation $(S=K \exp (x))$. Then with the Finite Difference method, we calculate the value of $u$. After that, the output $u$ of the finite difference becomes the input of our RBF network, so we train and then we simulate our input, to get finally a new output that we use to calculate the price of the put option in equation (2.6).

To test the reliability of our algorithm, we will present in the next section some numerical results of an American put option as an example.

\section{Numerical Experiments and Results}

This section consists in comparing the American put option value obtained by the Black Scholes (BS) model, using two approaches; Monte Carlo simulation and RBF neural network. The comparison will include the price of option, time performance, and sensitivity by calculating the delta hedging of the American put option.

4.1. Price American put Option using RBF network versus Monte Carlo. In order to improve the results obtained by RBF neural network, we chose to compare the price of the put American option that we get from the told method, and those obtained by the classic Monte Carlo method, with the semi-analytic results. 
TABle 1. American put option price: using Monte Carlo approach, finite difference and RBF network approach. with Strike $=55$, interest rate $=0.05, \mathrm{~T}=1$

\begin{tabular}{|c|c|c|c|c|c|}
\hline$S_{0}$ & $\sigma$ & \multicolumn{3}{|c|}{ Put Price } & \multirow{2}{*}{ Semi Analytic solution } \\
\cline { 3 - 5 } & & BS-Monte Carlo & BS-Finite difference & BS-Neural RBF & \\
\hline 50 & 0,2 & 5.9898 & 5.5977 & 5.4914 & 5.3377 \\
\hline 50 & 0,4 & 9.4796 & 9.4531 & 9.3736 & 9.3197 \\
\hline 52 & 0,2 & 4.7691 & 4.5120 & 4.4370 & 4.3155 \\
\hline 52 & 0,4 & 8.6586 & 8.5828 & 8.5140 & 8.4279 \\
\hline 54 & 0,2 & 3.6455 & 3.6286 & 3.5613 & 3.4459 \\
\hline 54 & 0,4 & 7.5555 & 7.7975 & 7.7370 & 7.6115 \\
\hline 56 & 0,2 & 2.9138 & 2.8896 & 2.8446 & 2.7190 \\
\hline 56 & 0,4 & 6.9494 & 7.0799 & 7.0294 & 6.8662 \\
\hline
\end{tabular}

In this study we supposed that the market conditions has been verified, so the table 1 described the different values of the put option while changing the value of the stock price (i.e. underlying asset) at $t=0$, and as we know the volatility has a big effect on the price equation, so we tried to fixed two values of volatility, to detect the impact from using two different methods to evaluate our option. Our method is based on the values obtained from finite difference, then we use the RBF network to reinforce the results in order to be more sophisticated and close to analytic solutions, which is clear from table (1). If we compare the results of those two methods, we can see that RBF network results are more close to the analytic solution than the finite difference values.

Moreover, as shown above, the values that we get from RBF network are close to the semianalytic solution more than Monte Carlo simulation, even if we have augmented the path into 1000 iterations to execute the Monte Carlo algorithm and, of course, to get a closer results and to minimize the error as possible. Otherwise, the RBF neural network algorithm path is limited to 150 simulation.

4.2. Comparing Monte Carlo and RBF neural network performance. CPU time (central processing unit), is one of the most important parameters that prove the performance of such a method (or a code). For this reason, we calculate the CPU time (in seconds) at each step of calculation of our price put option, and the error training as well

As shown in table (2), even if there is not a big difference between the speed of exercising of RBF neural network and Monte Carlo, the fact that we are working just in one dimension and still neural network seems to be faster than Monte Carlo method is a very interesting result, because if we moved to a high dimension machine learning, in the general case, it will has acceptability to solve the problem and here Monte Carlo faced the old issue which is the curse of dimensionality. So, the RBF neural network has the priority to work with since one of our goals is to speed up the calculation time to enhance the American option.

Other parameters that we assimilated, to improve the performance of our approach are summed square of residuals, R-square measure, and the Log-likelihood. 
TAble 2. Performance comparison: CPU and MSE. American put option, with Strike $=55$, interest rate $=0.05, \mathrm{~T}=1$

\begin{tabular}{|c|c|c|c|c|c|}
\hline \multirow{2}{*}{$S_{0}$} & $\sigma$ & \multicolumn{2}{|c|}{ CPU time (sec) } & \multicolumn{2}{c|}{ MSE training } \\
\cline { 3 - 6 } & & BS-Monte Carlo & BS-Neural RBF & BS-Monte Carlo & BS-Neural RBF \\
\hline 50 & 0,2 & 0.6373 & 0.5594 & 0.0331 & $4.2945 e^{-6}$ \\
\hline 50 & 0,4 & 0.6254 & 0.5686 & 0.0547 & $4.5291 e^{-7}$ \\
\hline 52 & 0,2 & 0.6220 & 0.5765 & 0.0137 & $7.0928 e^{-6}$ \\
\hline 52 & 0,4 & 0.6233 & 0.6280 & 0.0416 & $8.8540 e^{-7}$ \\
\hline 54 & 0,2 & 0.6322 & 0.5830 & 0.0187 & $5.6498 e^{-6}$ \\
\hline 54 & 0,4 & 0.7073 & 0.5624 & 0.0381 & $6.3635 e^{-7}$ \\
\hline 56 & 0,2 & 0.6700 & 0.5666 & 0.0146 & $4.3607 e^{-6}$ \\
\hline 56 & 0,4 & 0.6293 & 0.5719 & 0.0371 & $3.9006 e^{-7}$ \\
\hline
\end{tabular}

TABLE 3. Statistic value: SSE,R-square and Log likelihood of the put option

\begin{tabular}{|c||c|c|c|}
\hline Methods & SSE & R-square & Log likelihood \\
\hline Monte carlo - BS & 20,13 & 0,7849 & -177.438 \\
\hline RBF - neural netwrok & 14,4 & 0,9994 & $-550,371$ \\
\hline
\end{tabular}

As it is known, that summed square of residuals (SSE) measures the total deviation of the response values. The value obtained using the RBF neural network method is smaller than $\mathrm{MC}$ and closer to 0 , so the neural network is more useful for predict the price value. On other hand, R-square measures the degree of explaining the variation data called also the square of the multiple correlation coefficient, and the table shows that RBF neural network's R-square is more significant.

4.3. Comparing Monte Carlo and RBF neural network sensitivity. Hedging is a strategy used by investors and market actors to reduce their exposure to risk against undesirable events, thus the delta hedging establishing the required hedge that may be accomplished by buying or selling an amount of the underlying that corresponds to the delta of the portfolio.

To measure the sensitivity we calculate the delta parameter. Delta measures the amount to which the theoretical price of the option obtained by Black Scholes partial differential equation, will change if the market moves (up or down), in order to give the investor a better idea of how a stock has been performing. So it's a hedging strategy.

We can consider the delta hedging as a relationship between an option price, and the underlying stock price: $\frac{\partial V_{t}}{\partial S_{t}}$

We divided time interval ( $N$ steps) into 15 subinterval to calculate delta hedging 


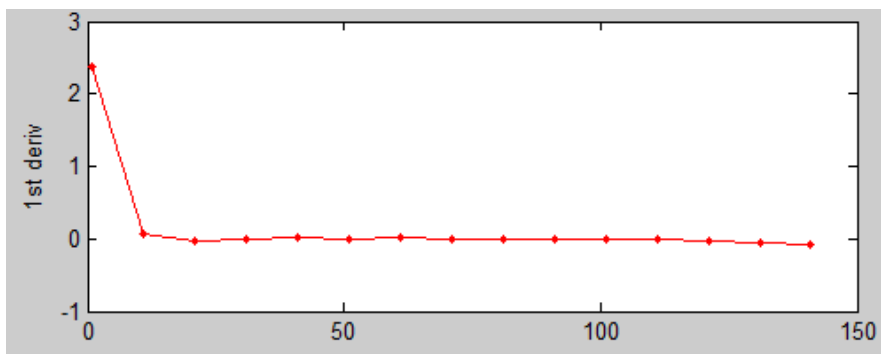

Figure 2. Delta hedging Monte Carlo-BS

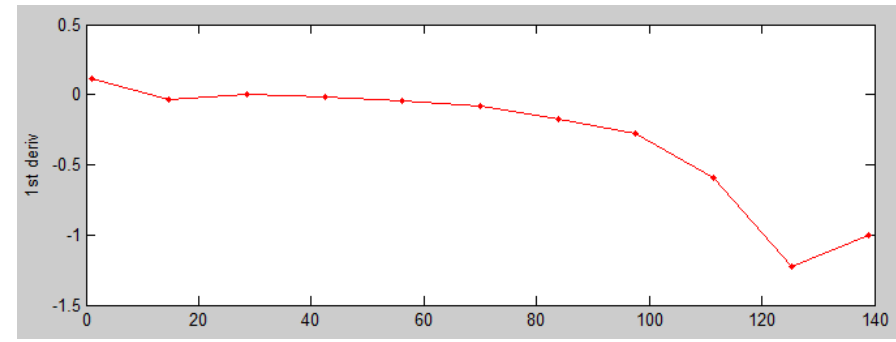

FIGURE 3. Delta hedging RBF neuarl network

The variation of the price according to the stock price or the delta hedging reached show's that RBF network delta is more interesting, taking for example $n=77$ (at month June because we have $T=1$ (year) and $N=150$ ), delta $a_{M C}=-0,0051$ that means that the option price expected to decreases in value in 0,0051 , and for the RBF neural network; delta $a_{\text {RBFNN }}=$ $-0,1017$.

\section{Note and Discussion}

In this work, we have proposed a neural network approach to price the American put option. We have combined the neural network to solve the Black Scholes partial differential equation with finite difference method using radial basis function. Our objective in the first place was to compare the performance and the results that we got from Monte Carlo simulation with radial basis function neural network. Even if the speed of convergence of Monte Carlo method was closer to the RBF-NN accelerating, the value obtained from neural network was closer to the semi-analytic solution.

The problem of dimensionality always exists when using Monte Carlo method, for this reason, several studies appear to reduce this problem (variance reduction, quasi-Monte Carlo, discrepancy sequences...). However, using neural networks leads to solving -somehow- this curse of dimensionality. Moreover, the speed of convergence will be increased by the number of training of our neural network algorithm. 


\section{References}

[1] Alanis, A. Y., Arana-Daniel, N., \& Lopez-Franco, C. (Eds.). Artificial Neural Networks for Engineering Applications. Academic Press (2019).

[2] Al-Aradi, A., Correia, A., Naiff, D., Jardim, G., \& Saporito, Y. Solving nonlinear and high-dimensional partial differential equations via deep Learning. arXiv preprint arXiv:1811.08782 (2018).

[3] Alqezweeni, M., \& Gorbachenko, V. Solution of Partial Differential Equations on Radial Basis Functions Networks (No. 1964). EasyChair (2019).

[4] Bennell, J., \& Sutcliffe, C. BlackScholes versus artificial neural networks in pricing FTSE 100 options. Intelligent Systems in Accounting, Finance \& Management: International Journal, 12(4), 243-260 (2004).

[5] Bhatia, G. S., \& Arora, G. Radial basis function methods for solving partial differential equations-A review. Indian Journal of Science and Technology, 9(45), 1-18 (2016).

[6] Black, F., \& Scholes, M. The valuation of options and corporate liabilities. Journal of Political Economy, 81(3), 637-654(1973).

[7] Bollig, E. F., Flyer, N., \& Erlebacher, G. Solution to PDEs using radial basis function finite-differences (RBF-FD) on multiple GPUs. Journal of Computational Physics, 231(21), 7133-7151 (2012).

[8] Brennan, M. J., \& Schwartz, E. S. Finite difference methods and jump processes arising in the pricing of contingent claims: A synthesis. Journal of Financial and Quantitative Analysis, 13(3), 461-474 (1978).

[9] Broomhead, D. S., \& Lowe, D. Radial basis functions, multi-variable functional interpolation and adaptive networks (No. RSRE-MEMO-4148). Royal Signals and Radar Establishment Malvern (United Kingdom) (1988).

[10] Chen, Y., \& Wan, J. W. Deep neural network framework based on backward stochastic differential equations for pricing and hedging american options in high dimensions. arXiv preprint arXiv:1909.11532 (2019).

[11] Clment, E., Lamberton, D., \& Protter, P. An analysis of a least squares regression method for American option pricing. Finance and Stochastics, 6(4), 449-471 (2002).

[12] Cortese, A., De Martino, B., \& Kawato, M. The neural and cognitive architecture for learning from a small sample. Current opinion in neurobiology, 55, 133-141 (2019).

[13] Dumitrescu, R., Quenez, M. C., \& Sulem, A. American options in an imperfect market with default. arXiv preprint arXiv:1708.08675 (2017).

[14] Ekstrm, E., \& Tysk, J. The BlackScholes equation in stochastic volatility models. Journal of Mathematical Analysis and Applications, 368(2), 498-507 (2010).

[15] El Karoui, N., \& Quenez, M. C. Non-linear pricing theory and backward stochastic differential equations. In Financial mathematics (pp. 191-246). Springer, Berlin, Heidelberg (1997).

[16] El Karoui, N., Pardoux, ., \& Quenez, M. C. Reflected backward SDEs and American options. Numerical methods in finance, 13, 215-231 (1997).

[17] Fu, M. C., Laprise, S. B., Madan, D. B., Su, Y., \& Wu, R. Pricing American options: A comparison of Monte Carlo simulation approaches. Journal of Computational Finance, 4(3), 39-88 (2001).

[18] Gurney, K. An introduction to neural networks. CRC press (2014).

[19] Hackmann, D. Solving the Black Scholes equation using a finite difference method. Available online: math. yorku. ca/ dhackman/BlackScholes7. pdf (2009).

[20] Hardy, R. L. Multiquadric equations of topography and other irregular surfaces. Journal of geophysical research, 76(8), 1905-1915 (1971).

[21] Higham, D. J. An introduction to financial option valuation: mathematics, stochastics and computation (Vol.

13). Cambridge University Press (2004).

[22] Jacquier, A. Numerical Methods in Finance. Lecture Notes, Imperial College London. Retrieved from https://www. imperial. ac. uk/on 20th November (2016).

[23] Janson, S., \& Tysk, J. FeynmanKac formulas for BlackScholes-type operators. Bulletin of the London Mathematical Society, 38(2), 269-282 (2006).

[24] Kasabov, N. K. Time-space, spiking neural networks and brain-inspired artificial intelligence. Heidelberg: Springer (2019).

[25] Kolesnikov, D. Numerical Methods for Pricing American Put Options (2015). 
[26] Liu, S., Oosterlee, C. W., \& Bohte, S. M. Pricing options and computing implied volatilities using neural networks. Risks, 7(1), 16 (2019).

[27] Longstaff, F. A., \& Schwartz, E. S. Valuing American options by simulation: a simple least-squares approach. The review of financial studies, 14(1), 113-147 (2001).

[28] Malliaris, M., \& Salchenberger, L. A neural network model for estimating option prices. Applied Intelligence, 3(3), 193-206 (1993).

[29] McNelis, P. D. Neural networks in finance: gaining predictive edge in the market. Academic Press, (2005).

[30] Medvedev, A., \& Scaillet, O. Pricing American options under stochastic volatility and stochastic interest rates. Journal of Financial Economics, 98(1), 145-159 (2010).

[31] Natenberg, S. Option volatility and pricing: Advanced trading strategies and techniques. McGraw-Hill Education (2014).

[32] Oksendal, B. Stochastic differential equations: an introduction with applications. Springer Science \& Business Media (2013).

[33] Papanicolaou, A. Introduction to Stochastic Differential Equations (SDEs) for Finance. arXiv preprint arXiv:1504.05309 (2015).

[34] Pham, H. Optimisation et contrle stochastique appliqus la finance (Vol. 61). Berlin: Springer (2007).

[35] Racicot, F. ., \& Thoret, R. Finance computationnelle et gestion des risques. PUQ (2006).

[36] Roncalli, T. (2001). Introduction la gestion des risques. Cours ENSAI, France. World Wide Web: http://www. univ-evry. fr/modules/resources/download/default/m2if/roncalli/gdr. pdf.(Accessed September 5, 2013).

[37] Rubinstein, R. Y., \& Kroese, D. P. Simulation and the Monte Carlo method (Vol. 10). John Wiley \& Sons (2016).

[38] Schwartz, E. S. The valuation of warrants: Implementing a new approach. Journal of Financial Economics, 4(1), (1977) 79-93.

[39] Smith, G. D., Smith, G. D., \& Smith, G. D. S. Numerical solution of partial differential equations: finite difference methods. Oxford university press (1985).

[40] Sodhi, A. American Put Option pricing using Least squares Monte Carlo method under Bakshi, Cao and Chen Model Framework (1997) and comparison to alternative regression techniques in Monte Carlo. arXiv preprint arXiv:1808.02791 (2018).

[41] peranda, I. \& Trinski, Z. Hedging as a business risk protection instrument. Ekonomski vjesnik: Review of Contemporary Entrepreneurship, Business, and Economic Issues, 28(2), (2015), 551-565.

[42] Sundaram, R. K. Derivatives in financial market development. International Growth Centre London, September. Pobrano z: http:/ / pages. stern. nyu. edu/ersundara (2012).

[43] Statistiques, D. E. A. \& Alatoires enEconomie, M.. Mthodes de Monte Carlo pour la finance (2004).

[44] Wilmott, P. Derivatives: The theory and practice of financial engineering. Wiley (1998).

[45] Wilmott, P. Paul Wilmott on quantitative finance. John Wiley \& Sons (2013).

[46] Xie, T., Yu, H. \& Wilamowski, B. Comparison between traditional neural networks and radial basis function networks. In 2011 IEEE International Symposium on Industrial Electronics (2011) (pp. 1194-1199). IEEE.

[47] Yadav, N., Yadav, A. \& Kumar, M. . An introduction to neural network methods for differential equations (pp. 13-15). Netherlands: Springer (2015). 